\title{
Variación temporal en la distribución geográfica y ecológica de Amazona finschi (Psittaciformes: Psittacidae)
}

\author{
Alejandro Sánchez-Barradas ${ }^{1}$, Quiyari J. Santiago-Jiménez² \& Octavio Rojas-Soto ${ }^{3 *}$ \\ 1,2. Museo de Zoología, Facultad de Biología-Xalapa, Universidad Veracruzana, Zona Universitaria, Circuito Gonzalo \\ Aguirre Beltrán S/N, Xalapa, Veracruz, C.P. 91090, MÉXICO; alex-sb@hotmail.com, qsantiago@uv.mx \\ 3. Red de Biología Evolutiva, Laboratorio de Bioclimatología, Instituto de Ecología A. C. km 2.5 Antigua Carretera a \\ Coatepec no. 351, Xalapa 91070, Veracruz, México; octavio.rojas@inecol.mx \\ * Correspondence
}

Recibido 11-XI-2016. Corregido 02-III-2017. Aceptado 03-IV-2017.

\begin{abstract}
Temporal variation in ecological and geographical distribution of Amazona finschi (Psittaciformes: Psittacidae). The lilac-crowned parrot (Amazona finschi) is an endemic species restricted to lowlands of the Mexican Pacific coast and is currently considered as endangered. It has been documented that it shows altitudinal and seasonal migrations along its distributional range, suggesting that its ecological and temporal distribution is still uncertain. We modeled the potential distribution of the $A$. finschi considering the two main activity seasons for the species: reproductive and non-reproductive. We used 428 historical occurrences (1882-2014), obtained from open access databases, combined with five environmental layers: three climatic and two topographic for each season, to perform ecological niche models using Maxent. We then transferred each model to the rest of the months to analyze the seasonal movements guided by climate. Differences in ecological variables between seasons were evaluated using a t-test. The geographic correspondence among the parrot distribution and the resources (plants) distributions were analyzed by superimposing maps. A marked seasonality in the distribution of $A$. finschi was observed. Ecologically, the species displays greater amplitude during the breeding seasons in terms of minimum temperature, but a noticeable reduction as far as precipitation is concerned. The distribution of food and nesting resources largely corresponds to the distribution of this parrot. There is a wide area in the center of the geographical distribution in which the species finds conditions that meet both periods of activity, as well as transition conditions between these periods. Finally, our results on the seasonal variation in the geographical and ecological distribution of this species, possess a strong ecological meaning in the understanding of other species distribution, particularly those associated with highly seasonal environments, and definitively will contribute to the conservation of this species. Rev. Biol. Trop. 65 (3): 1194-1207. Epub 2017 September 01.
\end{abstract}

Key words: breeding season, modelling, ecological niche, endangered, nest, diet, coexistence.

La variación climática es un fenómeno importante en ambientes tropicales ya que las diferencias en los períodos de lluvias pueden causar una marcada estacionalidad (Sarmiento, 1972; Loiselle \& Blake, 1994). Estas fluctuaciones a su vez, pueden influenciar la estructura del hábitat y la disponibilidad de los recursos (Malizia, 2001; Codesido \& Bilenca, 2004). A causa de lo anterior, la interacción de las especies con su medio a lo largo del año puede ser variable (Wiens, 1989; Loiselle \& Blake, 1994;
Codesido \& Bilenca, 2004). Por otro lado, la estacionalidad no solo afecta la interacción de las especies con el hábitat, sino que también influye en la fisiología y conducta de las especies y en último caso, en la coexistencia entre estas (Anderson, 1973; Leitner, Voight \& Gahr, 2001; Liknes \& Swanson, 2011).

Un claro ejemplo del efecto de la estacionalidad son los patrones de distribución temporal de las especies (Stiles, 1979; Hilty, 1997). Dichos patrones nos ayudan a entender 
de qué manera las poblaciones residentes se ajustan a la fluctuación en la disponibilidad de recursos, dada por las variaciones ambientales (Karr, 1976; Herrera, 1978; Rabenold \& Rabenold, 1985; Loiselle \& Blake, 1991). A pesar de lo anterior, la mayoría de los esfuerzos dedicados a entender el fenómeno migratorio en aves, se han concentrado en las causas y patrones de movimientos migratorios de varios miles de kilómetros (Boyle \& Conway, 2007; Boyle, 2008).

No solo los movimientos migratorios intratropicales, altitudinales o migraciones parciales, permanecen aún poco entendidos (Sekercioglu, 2010), también el efecto de la estacionalidad en la distribución geográfica de aves ha sido poco estudiado (La Sorte et al., 2015), particularmente en grandes escalas geográficas. Un claro ejemplo es el del loro corona lila, Amazona finschi (Sclater, 1864), especie endémica de México, que se distribuye en las tierras bajas de la vertiente del Pacífico, y asociada a las selvas secas desde Sonora hasta el Istmo de Tehuantepec (Forshaw, 1989; Howell \& Webb, 1995; Ortega-Rodríguez \& Monterrubio-Rico, 2008; Ríos-Muñoz \& Navarro-Sigüenza, 2009; Marín-Togo et al., 2012). Se ha reportado que $A$. finschi realiza tanto migraciones altitudinales como regionales en respuesta a las variaciones estacionales del clima (de la Parra-Martínez, 2011); por lo anterior, el loro corona lila puede ser un excelente modelo del efecto de la variación ambiental en la distribución temporal de las especies.

El Pacífico mexicano se caracteriza por una alta variación estacional con grandes diferencias en la precipitación entre temporadas (Trejo \& Dirzo, 2000). Asimismo, se ha observado que dicha estacionalidad ejerce presión en la reproducción de $A$. finschi, (Renton, 2001, 2002; Renton \& Íñigo-Elías, 2003; Renton \& Salinas-Melgoza, 2004; Salinas-Melgoza \& Renton, 2005; Ortega-Rodríguez \& Monterrubio-Rico, 2008; Álvarez, 2010; de la Parra Martínez 2011), así como en su distribución (Renton \& Íñigo-Elías, 2003). Un claro ejemplo es el rango altitudinal de la especie, reportado desde el nivel del mar hasta los 2000 metros (Friedmann, Griscom, \& Moore, 1950; Forshaw, 1989; Stotz, Fitzpatrick, Parker, \& Moskovits, 1996), pero que a partir del 2002 se ha reducido alrededor de los $1000 \mathrm{msnm}$, siendo muy raro encontrar a la especie por encima de esta cota (Renton \& Íñigo-Elías, 2003). A causa de lo anterior se ha observado una mayor abundancia de la especie entre los 500 y 600 msnm (Renton \& Íñigo-Elías, 2003; Álvarez, 2010).

Hasta los años 70, al loro corona lila se le consideraba común a lo largo de su distribución (Ridgely, 1981). Sin embargo, debido a su vulnerabilidad intrínseca, el tráfico ilegal y la pérdida de hábitat, las poblaciones de la especie se han visto severamente afectadas (Masera, Ordoñez, \& Dirzo, 1997; Trejo \& Dirzo, 2000; Renton \& Íñigo-Elías, 2003; Marín-Togo et al., 2012). Por lo anterior, la especie pasó de ser catalogada como "amenazada" a "en peligro", tanto por la Norma Oficial Mexicana NOM059 (D.O.F., 2010), como por la Unión Internacional para la Conservación de la Naturaleza (UICN; http://www.iucnredlist.org).

Los modelos de nicho ecológico (MNE), dada su versatilidad, han sido usados para evaluar prioridades de conservación en aves en peligro de extinción (Papes, 2007). Igualmente, han resultado muy útiles para explorar movimientos migratorios en aves, tanto de varios miles de kilómetros (Nakazawa et al., 2004, Pérez-Moreno et al., 2016), como movimientos migratorios cortos de cientos de kilómetros (Sobral-Macondes, Del-Río, Rego \& Silveira, 2014). La utilidad de los MNE radica en la relación que existe entre la fisiología y las tasas de crecimiento poblacional de las especies (Soberón, 2007), lo que al final determina la presencia o ausencia de una especie en un espacio dado.

Actualmente, no existen estudios enfocados al análisis de la distribución estacional del loro corona lila, que consideren toda su área de distribución y su variación climática, la cual ejerce una gran presión fisiológica sobre el loro, particularmente durante su periodo reproductivo (Renton \& Salinas-Melgoza, 2004). A la fecha, el único trabajo que incluye 
toda el área de distribución es el de Renton e Íñigo-Elías (2003), aunque sólo contempla la presencia y abundancia de la especie, sin considerar la estacionalidad. Si bien existen estudios que abordan la variación estacional en los requerimientos de la especie, como son: la dieta, la anidación y los parámetros reproductivos (Renton 2001, 2002; Renton \& Íñigo-Elías, 2003; Ortega-Rodríguez \& Monterrubio-Rico 2008; Álvarez, 2010), estos se encuentran en una escala que no permite el establecimiento de estrategias de conservación globales para la especie. Por lo anterior, el objetivo del presente trabajo fue describir la distribución geográfica, ecológica y estacional del $A$. finschi mediante el uso de MNE.

\section{MATERIAL Y MÉTODOS}

Obtención de información puntual y ambiental: Se generó una base de datos a partir de registros de ocurrencia de la especie procedentes de distintas colecciones (Apéndice digital 1), los cuales fueron obtenidos, a partir de la Infraestructura Mundial de Información sobre Biodiversidad (Global Biodiversity Information Facilities, GBIF por sus siglas en inglés). Se incluyeron todos los registros desde 1882 hasta 2014, aunque estos se ubicaran en áreas donde la especie no ha sido registrada desde 2002 (Oaxaca; Renton \& Íñigo-Elías, 2003), ya que estos contienen información ambiental importante. Todos los registros de ocurrencia se analizaron en un sistema de información geográfica (SIG) ArcGis 10.1 (ESRI, 2011); se eliminó aquellos mal georreferenciados, sin fecha, duplicados y dudosos, considerando la distribución histórica conocida de la especie (Howell \& Webb, 1995; Navarro-Sigüenza \& Peterson, 2007), y se obtuvo una base de datos verificada de 428 presencias históricas. Posteriormente, los datos se separaron por mes (35 presencias en promedio, con un rango de 10 a 64 y una desviación estándar de 19) y por temporada tomando en cuenta lo propuesto por Renton y Salinas-Melgoza (1999): reproductiva (febrero a mayo, 208 presencias) y no reproductiva (junio a octubre, 106 presencias).
Para caracterizar el nicho ambiental se usaron coberturas correspondientes a las temperaturas mínimas y máximas, así como la precipitación acumulada por mes (Hijmans, Cameron, Parra, Jones \& Jarvis, 2005), las cuales se encuentran a una resolución de 0.0083 grados $\left(\sim 1 \mathrm{~km}^{2}\right)$ y son el promedio de 50 años. Se incluyeron además dos coberturas topográficas: el índice topográfico compuesto (CTI; que se refiere a la capacidad del terreno para encharcar el agua considerando su forma) y la pendiente (USGS, 2005), que son variables determinantes para la especie (Ortega-Rodríguez \& Monterrubio-Rico, 2008).

La influencia de las variables y la validación de los modelos dependen de la extensión del área accesible a la especie; es decir, el área histórica donde la especie se circunscribe (Soberón, 2007; Barve et al., 2011). Para calcular dicha área, se generó una zona de amortiguamiento que contuviera la totalidad de los registros de la especie, usando como referencia el desplazamiento máximo conocido del loro corona lila que es de $50 \mathrm{~km}$ (Renton \& SalinasMelgoza, 2004). Como resultado, se obtuvo un polígono en el cual se incluyó todo lo que estuviera en un radio de dos y media veces el desplazamiento máximo conocido para la especie $(125 \mathrm{~km})$ para cada punto. Si bien, las zonas de amortiguamiento no consideran aspectos topográficos, nos facilitan establecer mediante el modelado, las áreas climáticamente óptimas en las que la especie puede habitar.

Modelado: El desarrollo de los MNE se llevó a cabo mediante el uso de MaxEnt (ver. 3.3.3k, Phillips, Anderson, \& Schapire, 2006) (Apéndice digital 1). Para la parametrización de cada ejercicio de modelado, se mantuvieron los valores por defecto de MaxEnt, pero se desactivaron las opciones de "do clamping" y "extrapolate" para evitar extrapolaciones artificiales en los valores extremos de las variables ecológicas. Para cada modelo se realizaron cinco réplicas, de las cuales se conservó la que presentó el valor más bajo de omisión para los datos de validación. Se usó la salida logística para obtener mapas digitales 
con probabilidades de idoneidad de hábitat continuas con valores de 0 a 1 (Phillips et al., 2006). Para transformar los mapas probabilísticos a binarios, considerando que los datos provienen de fuentes con distintos de grados de certeza, se estableció un umbral del $10 \%$ de omisión en los puntos de entrenamiento; es decir, se estableció como presente (1) a partir del valor de probabilidad correspondiente con la inclusión del $90 \%$ de los puntos de entrenamiento y como ausente $(0)$ ante los valores más bajos de ese umbral.

La validación de los modelos se llevó a cabo mediante dos aproximaciones, una a través de los valores del área bajo la curva (AUC por sus siglas en inglés) de la curva ROC (Receiver Operating Characteristic) para los modelos mensuales. Sin embargo, debido a que diversos problemas han sido asociados a esta prueba (Peterson, Papes \& Soberón, 2008; Lobo, Jiménez-Valverde \& Real, 2008), se usó una modificación llamada ROC parcial, la cual otorga un peso diferencial a los errores de omisión y comisión (Peterson et al., 2008). Esta prueba se utilizó solo para los modelos estacionales mediante el programa "Tool for Partial-ROC V. 1.0." (Barve, 2008) y se consideró un valor de error de omisión menor a 5 $\%$. Para los modelos mensuales solo se usó la ROC normal ya que se contaba con muy pocos registros ( 30). Para medir el desempeño de los modelos, tanto en la ROC normal como en el caso de la ROC parcial, se usó el $20 \%$ de los puntos para su validación.

Los modelos generales de las temporadas reproductiva y no reproductiva se generaron utilizando la temperatura máxima y mínima, provenientes de las coberturas mensuales correspondientes, para así obtener el rango más amplio de temperatura de cada temporada. Esto se llevó a cabo, al seleccionar sólo las coberturas de los meses que incluyeran los datos máximo y mínimo de temperatura, dentro del conjunto de meses incluidos en cada temporada. Además, se incluyó el promedio de la precipitación mensual de cada temporada, el cual se realizó en ArcGIS mediante una operación de algebra de mapas que consistió en la suma de todos los mapas que componen la temporada, para luego dividir el total resultante entre el número de mapas sumados. Las variables topográficas (pendiente y CTI), al no presentar variación mensual, se incluyeron sin modificaciones para ambas temporadas.

Los modelos estacionales fueron proyectados (transferidos), al resto de los meses del año para evaluar la relación entre la distribución mensual de la especie y las condiciones de cada temporada, para así determinar si realmente existía una separación entre ambos periodos. Las condiciones de la temporada reproductiva se proyectaron a cada uno de los meses de junio a enero (meses correspondientes a la temporada no reproductiva); mientras que las condiciones de la temporada no reproductiva se proyectaron a cada uno de los meses de febrero a mayo (meses correspondientes a la temporada reproductiva). La delimitación de las temporadas se basó en lo propuesto por Renton (2009), mientras que la selección de las variables obedece a la importancia de estas de acuerdo a estudios previos (Ortega-Rodríguez \& Monterrubio-Rico, 2008; Álvarez, 2010; de la Parra-Martínez, 2011) y a que el resto de las variables bioclimáticas son promedios, rangos y coeficientes generales anuales (Hijmans et al., 2005), por lo cual no podían usarse en el presente estudio. Finalmente, para observar si las condiciones ambientales de una temporada estaban presentes en las condiciones de la otra temporada, se proyectaron las condiciones de la temporada reproductiva a la no reproductiva y viceversa.

Para analizar la variación climática estacional se extrajeron 20000 puntos al azar de los mapas de distribución potencial de cada temporada, y se obtuvieron sus valores para temperatura máxima y mínima y precipitación. Para probar la normalidad de los datos se usó una prueba de Kolmogorov-Smirnoff, y para determinar si existían diferencias entre las variables de cada temporada se realizaron pruebas de $\mathrm{t}$ student.

Dieta y sitios de anidación: Debido a que en escalas finas, algunos aspectos bióticos 
suelen influir en la distribución de las especies, para la temporada reproductiva se consideró a la presencia de las especies vegetales que el loro corona lila consume y aquellas en donde anida. Para esto se usaron los mapas de distribución elaborados por Pennington y Sarukhán (2005), para algunas de las especies que componen la dieta de A. finschi, como son Astronium graveolens, Brosimum alicastrum, Caesalpinia eriostachys y Caelenodendron mexicanum, y que de acuerdo a Renton (2001), estas representan más del $80 \%$ de los recursos alimenticios utilizados por la especie. Del mismo modo, se incluyeron las especies más usadas como sitios de anidación de acuerdo a Ortega-Rodríguez y Monterrubio-Rico (2008), como es el caso de Astronium graveolens, Brosimum alicastrum, Caelenodendron mexicanum y Enterolobium cyclocarpum. Mediante la suma de los mapas antes mencionados, se construyeron mapas de riqueza de recursos tanto alimenticios como de anidación. El análisis de dichos mapas nos permitió identificar aquellas áreas donde existe una mayor correspondencia geográfica de recursos que son fundamentales para la especie durante la etapa reproductiva (Renton \& Salinas-Melgoza, 1999; Renton, 2001; OrtegaRodríguez \& Monterrubio-Rico, 2008; Monterrubio-Rico, Ortega-Rodríguez, Marín-Togo, Salinas-Melgoza \& Renton, 2009). Si bien las especies vegetales extienden su distribución en otras regiones del país, como la Sierra Madre Oriental y la planicie del Golfo, cabe mencionar que el análisis sólo se realizó para el oeste de México, donde se distribuye $A$. finschi.

\section{RESULTADOS}

Distribución geográfica potencial: $\mathrm{La}$ distribución potencial del loro corona lila, generada con base en las condiciones climáticas mensuales, muestra que la región central del rango de distribución de la especie desde el sur de Sinaloa hasta Michoacán, presenta condiciones ambientales adecuadas para la presencia de la especie durante gran parte del año (Fig. 1). Los valores de la ROC para este periodo fueron

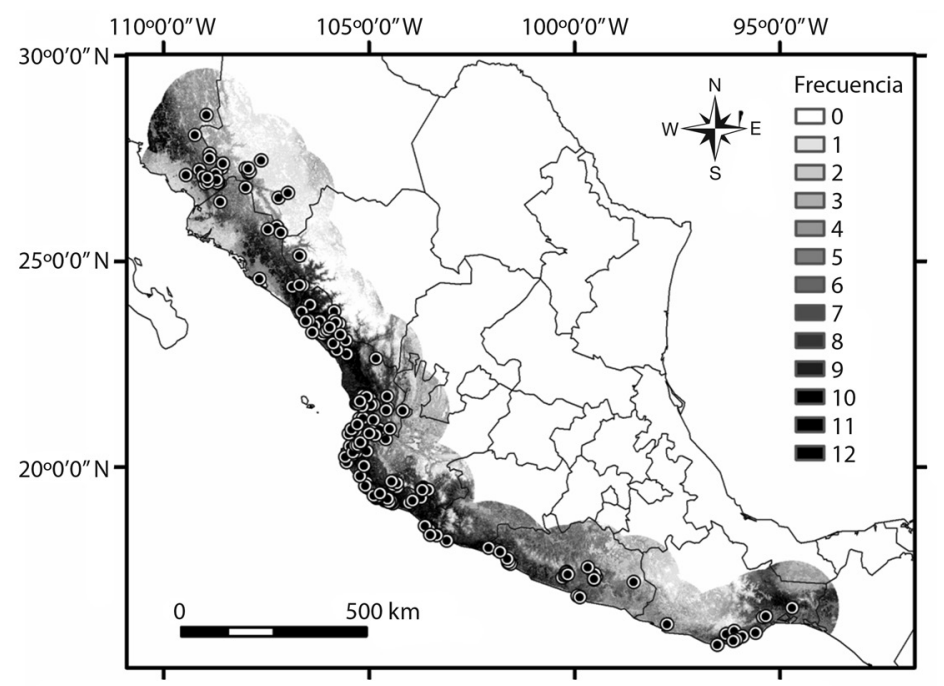

Fig. 1. Sumatoria de las distribuciones potenciales mensuales de Amazona finschi. La escala de grises indica la sumatoria de las predicciones de los modelos mensuales, que indican la coincidencia de las condiciones ambientales óptimas para la presencia de la especie a lo largo del ciclo anual, siendo 0 ausencia y 1-12 presencia durante el año. Los puntos indican la localidad de ocurrencia de la especie.

Fig. 1. The monthly potential distributions sum of Amazona finschi. The grayscale illustrates the sum of the predictions of the monthly models, which indicate the coincidence of the optimal environmental conditions for the presence of the species throughout the annual cycle, being 0 absence and 1-12 presence during the year. The points indicate the localities of occurrence of the species. 
en promedio de 0.83 , con un rango de 0.63 a 0.98 y una desviación estándar de 0.1 .

La distribución potencial de la especie se presenta de manera fragmentada en los estados de Guerrero y Oaxaca, donde las condiciones en las que la especie se desarrolla se presentan únicamente durante uno o dos meses al año (Fig. 1). Asimismo, muchas áreas del sur de la distribución, donde las condiciones se reducen, coinciden con sitios donde la especie ha sido extirpada. Por otro lado, el modelado demostró condiciones climáticas adecuadas para la especie durante la mayor parte del año en áreas donde el loro corona lila no ha sido registrado, como es el caso del noroeste de Chiapas (Fig. 1).

Durante la temporada reproductiva, se predijo una mayor área potencial hacia el centro del rango de la especie, en los estados de Sinaloa, Jalisco, Michoacán, Colima y Nayarit; así como una reducción y fragmentación notables en el sur, principalmente en el estado de Oaxaca (Fig. 2A). Por su parte, la distribución potencial del loro corona lila es ligeramente más amplia en la temporada de lluvias, presentando una mayor extensión hacia el sureste del país (Fig. 2B). Los valores producto de la ROC parcial para la temporada reproductiva fueron de un AUC ratio de $1.156(\mathrm{p}<0.001)$ y de $1.160(p<0.001)$ para la no reproductiva.

Variación estacional potencial: Las variables climáticas en sitios de presencia de los loros, se ajustaron a una distribución normal de acuerdo a la prueba KolmogorovSmirnoff $(\mathrm{p}<0.01)$, y presentaron diferencias significativas entre temporadas reproductivas y no reproductivas en temperatura mínima $(\mathrm{t}=$ -192.045, p < 0.001, g.1. 39 998), y precipitación $(\mathrm{t}=-401.174 \mathrm{p}<0.001$, g.l. 39 998; Fig. 2). Para la temperatura máxima y las variables topográficas, no se encontraron diferencias.

En cuanto a la variación mensual, la temperatura máxima tiende a mantenerse constante entre los 25 y 35 grados a lo largo de todo el año. Por su parte, la temperatura mínima presenta valores entre los 10 y 15 grados desde el mes de noviembre hasta el mes de mayo, mientras que de junio a octubre, se mantiene entre los 20 y 25 grados (Fig. 3). La precipitación presenta un patrón semejante al de la temperatura mínima, con valores menores a los 50 $\mathrm{mm}$ durante el periodo de noviembre a mayo, $\mathrm{y}$ valores iguales o mayores a los $100 \mathrm{~mm}$ durante los meses de junio a octubre (Fig. 3).

La distribución de la especie cuando considera sólo dos dimensiones ecológicas (temperatura máxima y temperatura mínima), muestra una ligera sobreposición entre las temporadas reproductiva y no reproductiva en las temperaturas cercanas a los $10^{\circ} \mathrm{C}$ y superiores para temperatura mínima, y mayores a los $25{ }^{\circ} \mathrm{C}$ para temperatura máxima (Fig. 4A). En cuanto a temperatura mínima y precipitación, ambas temporadas se separaron de manera notoria, ya que la temporada de reproducción se ubica entre los 0-50 $\mathrm{mm}$ aproximadamente, mientras que la temporada no reproductiva se expande desde alrededor de los 100 hasta poco más de $400 \mathrm{~mm}$ (Fig. 4B). Por otra parte, para la temperatura mínima, el rango fue más amplio en la temporada reproductiva $\left(3-22^{\circ} \mathrm{C}\right)$, y más estrecho en la no reproductiva $\left(9-24^{\circ} \mathrm{C}\right)$. La distribución ecológica considerando la precipitación y la temperatura máxima (Fig. 4C), muestra que en la temporada reproductiva, la especie está limitada por la precipitación y que hace uso de un mayor rango de temperatura máxima; en contraste, en la temporada no reproductiva, la especie usa sitios más cálidos reduciendo su rango en temperatura máxima y ampliando su rango en la precipitación.

La temperatura mínima y la precipitación de la especie se comportan de manera similar, con valores bajos en los meses de enero a mayo; se inicia un incremento durante este último mes, y se mantiene hasta octubre o noviembre. Por su parte, la temperatura máxima no obedece a este patrón, ya que presenta únicamente valores máximos en los meses de junio, julio y septiembre, con una disminución en agosto (Fig. 2). Al proyectar las condiciones de la temporada de reproducción al resto del año, se observó que estas sólo se presentan en los meses posteriores a la temporada de lluvias y previos al inicio de la temporada de secas 

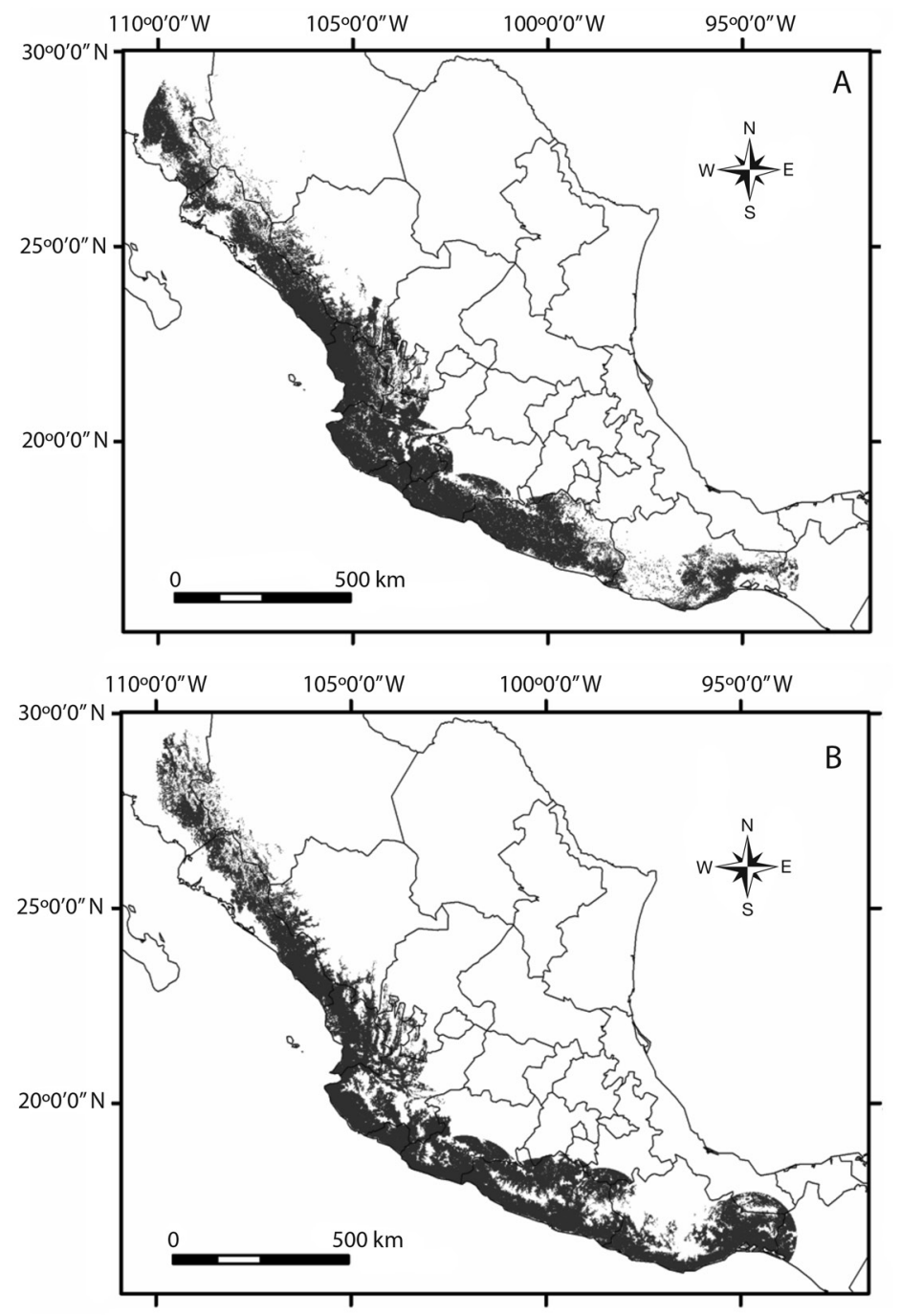

Fig. 2. Distribución geográfica potencial de A. finschi para la temporada de secas o reproductiva (A) y para la temporada de lluvias o no reproductiva (B).

Fig. 2. Potential geographical distribution of $A$. finschi for the dry or reproductive season (A) and for the rainy season or non-breeding season (B).

(octubre a enero). La proyección de lluvias al resto del año presenta un área constante en el sur del país en los estados de Oaxaca y Chiapas, cuya mayor extensión se observa en el mes de mayo (Apéndice digital 2).

\section{Correspondencia con riqueza de recur-} sos: Los patrones geográficos observados para la temporada reproductiva del loro corona lila, sugieren que las especies que sirven como alimento (y/o) sitio de anidación, coinciden de manera notable con la distribución potencial de loro (Fig. 5A y Fig. 5B). La distribución de las especies que sirven como alimento al loro corona lila presentan una distribución continua, observándose una correspondencia con las selvas secas de la Planicie Costera del Pacífico (Fig. 5A). Por su parte, la distribución 


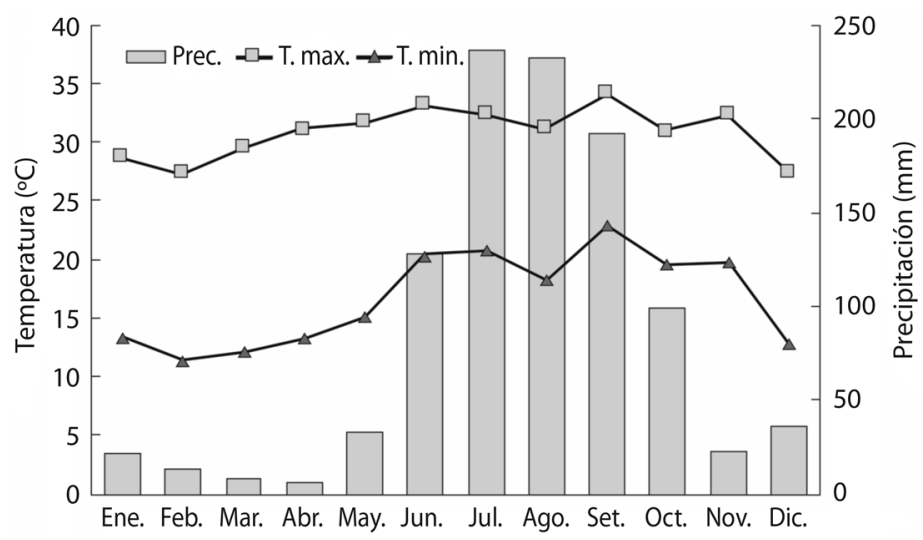

Fig. 3. Variación mensual en las temperaturas máximas y mínimas, así como en la precipitación de las que $A$. finschi hace uso a lo largo de los distintos meses del año.

Fig. 3. Monthly variation in the maximum and minimum temperatures, as well as in the precipitation that $A$. finschi uses during the different months of the year.

de las especies usadas para anidar presentan un patrón semejante, sólo que ocupando una mayor área en los estados de Oaxaca, Guerrero y Michoacán (Fig. 5B).

Al analizar la correspondencia con las especies de las que el loro corona lila se alimenta, estas presentan correspondencias variables que van desde un $6 \%$ (Caelenodendron mexicanum), hasta un $46 \%$ (Caesalpinia eriostachys). La única zona en la que coinciden las cuatro especies de árbol de las que la especie se alimenta se ubica en la zona centro de la distribución del loro (Fig. 5A), y corresponde a menos del $3.7 \%$ del área de distribución potencial para la temporada reproductiva. Para el caso de los árboles usados por la especie para anidar, la mayor correspondencia se presenta con la especie Enterolobium cyclocarpum (61\%), mientras que la menor correspondencia se da con la especie Caelenodendron mexicanum $(6 \%)$. El área de coincidencia entre las cuatro especies usadas para anidar representa el $3.9 \%$ de la distribución para la temporada reproductiva.

\section{DISCUSIÓN}

La distribución potencial de $A$. finschi varía entre temporadas en respuesta a las variaciones en el ambiente, siendo determinante la precipitación, lo que coincide con lo observado en otras especies (Sarmiento, 1972; Loiselle \& Blake, 1994). Debido a que estas fluctuaciones pueden modificar la estructura del hábitat y la disponibilidad de recursos (Malizia, 2001; Codesido \& Bilenca 2004), especies con un alto grado de sensibilidad, pueden presentar un bajo éxito reproductivo, como es el caso del loro corona lila (Renton \& Íñigo-Elías, 2003; Renton, 2009; de la Parra-Martínez, 2011). Estos cambios estacionales pueden, además, ocasionar variaciones en los patrones de distribución geográfica y ecológica de las especies (Stiles, 1978; Hilty, 1997), lo cual podría ser la causa por la cual la especie no se distribuye por encima de los 1000 msnm desde 2002 (Renton \& Íñigo-Elías, 2003). Lo anterior concuerda con lo reportado por Ortega-Rodríguez y Monterrubio-Rico (2008), quienes únicamente registraron el $10 \%$ de los nidos de la especie por encima de los 600 metros.

La especie no ha sido registrada en Chiapas a pesar de que esa zona cuenta con condiciones bajo las cuales la especie podría desarrollarse; si bien la especie ha atravesado el Istmo de Tehuantepec, es posible que este limite su dispersión al sur, como se ha observado para otras especies (García-Trejo \& NavarroSigüenza, 2004). Por otra parte, la presencia de Amazona autumnalis (Linnaeus, 1758), especie 

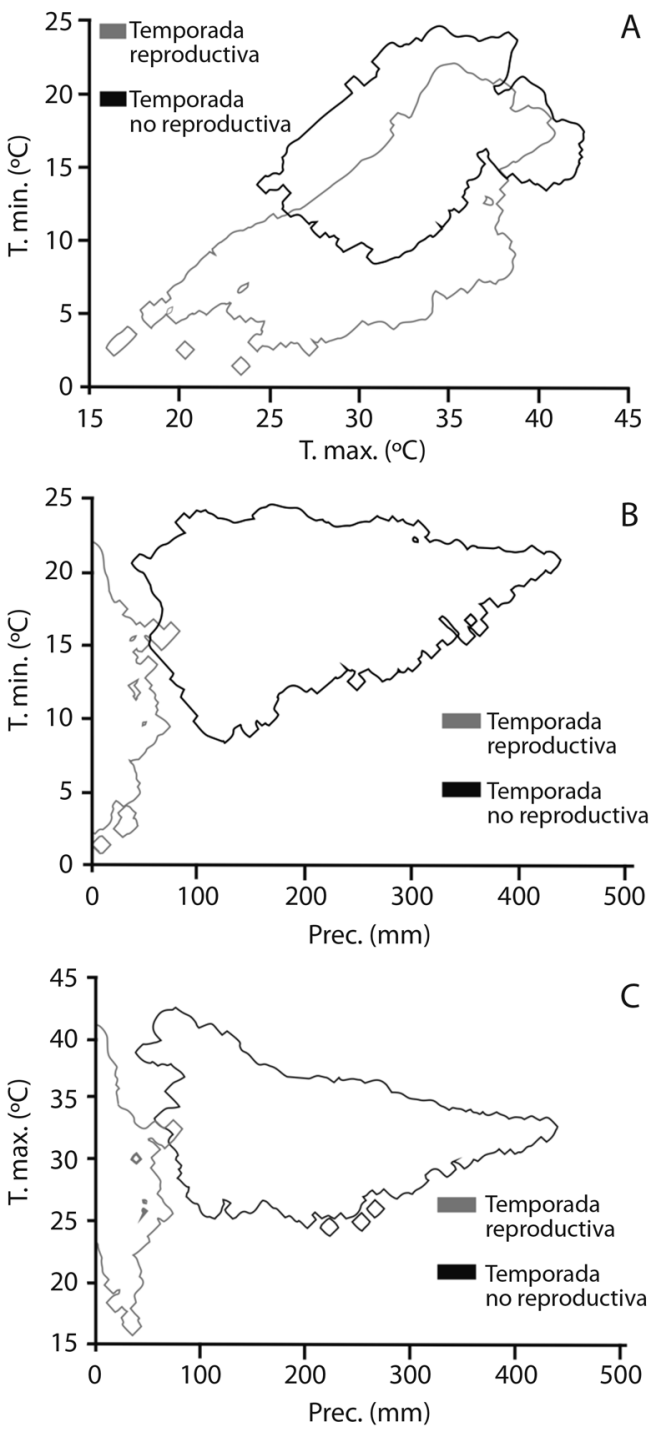

Fig. 4. Distribución estacional por temporadas (reproductiva y no reproductiva) de $A$. finschi con base en dos variables ambientales: la temperatura máxima y la temperatura mínima (A); la precipitación y la temperatura mínima (B) y la precipitación y la temperatura máxima $(\mathrm{C})$.

Fig. 4. Seasonal distribution by season (reproductive and non-reproductive) of $A$. finshi based on two environmental variables: maximum and minimum temperature $(\mathrm{A})$; the precipitation and the minimum temperature (B) and the precipitation and the maximum temperature $(\mathrm{C})$.

filogenéticamente muy relacionada a $A$. finschi y que pertenece al mismo gremio alimenticio (Russello \& Amato, 2004; Gómez de Silva et al., 2005b), podría impactar negativamente al loro corona lila a causa de la competencia entre ambas. Finalmente, el recambio en la vegetación (Rzedowski, 1978), y el aumento en la diversidad de especies de aves (NavarroSigüenza et al., 2014), pueden ser factores que en conjunto con el bajo éxito de anidación y productividad reproductiva del loro corona lila, limiten su distribución hacia el sur (Renton \& Ínigo-Elías, 2003).

Gracias a su alto grado de flexibilidad en la dieta (Renton, 2001), el loro corona lila puede reproducirse en la temporada seca a pesar de la limitada disponibilidad de recursos (Renton, 2001). Esto puede deberse a que durante esta temporada se reduce el gasto energético del proceso de reproducción y crianza, producto de la exposición a temperaturas y precipitación menores, lo cual se observa en la mayor amplitud de rango para temperatura mínima durante esta temporada y la escasa precipitación. Lo anterior puede estar relacionado con la sensibilidad de la especie y las condiciones propias de las cavidades usadas para anidar (Renton \& Íñigo-Elías, 2003; Ortega-Rodríguez \& Monterrubio-Rico, 2008), o bien ser una repercusión filogenética, ya que otras especies del género como A. albifrons (Sparman, 1788), у A. autumnalis se reproducen durante la misma temporada (Gómez de Silva, Oliveras \& Medellín, 2005a, b). En contraste con lo anterior, durante la temporada no reproductiva se presentaron rangos de temperatura máxima y mínima reducidos, que pueden estar relacionados con la distribución de los recursos importantes para que las hembras alcancen un óptimo metabólico previo a la temporada de crianza, así como para garantizar el éxito y supervivencia de la nidada (Selman \& Houston, 1996; Robb et al., 2008; Sorensen, Hipfner, Kyser \& Norris, 2009).

La fragmentación de hábitat influye en la presencia de las especies (Marín-Togo et al., 2012), por lo que sería preponderante analizar qué porcentaje del área aún cuenta con cobertura vegetal durante el periodo reproductivo del loro corona lila. En este estudio, debido a que la aproximación es macroclimática, no se consideró el cambio de uso de suelo; sin embargo, 

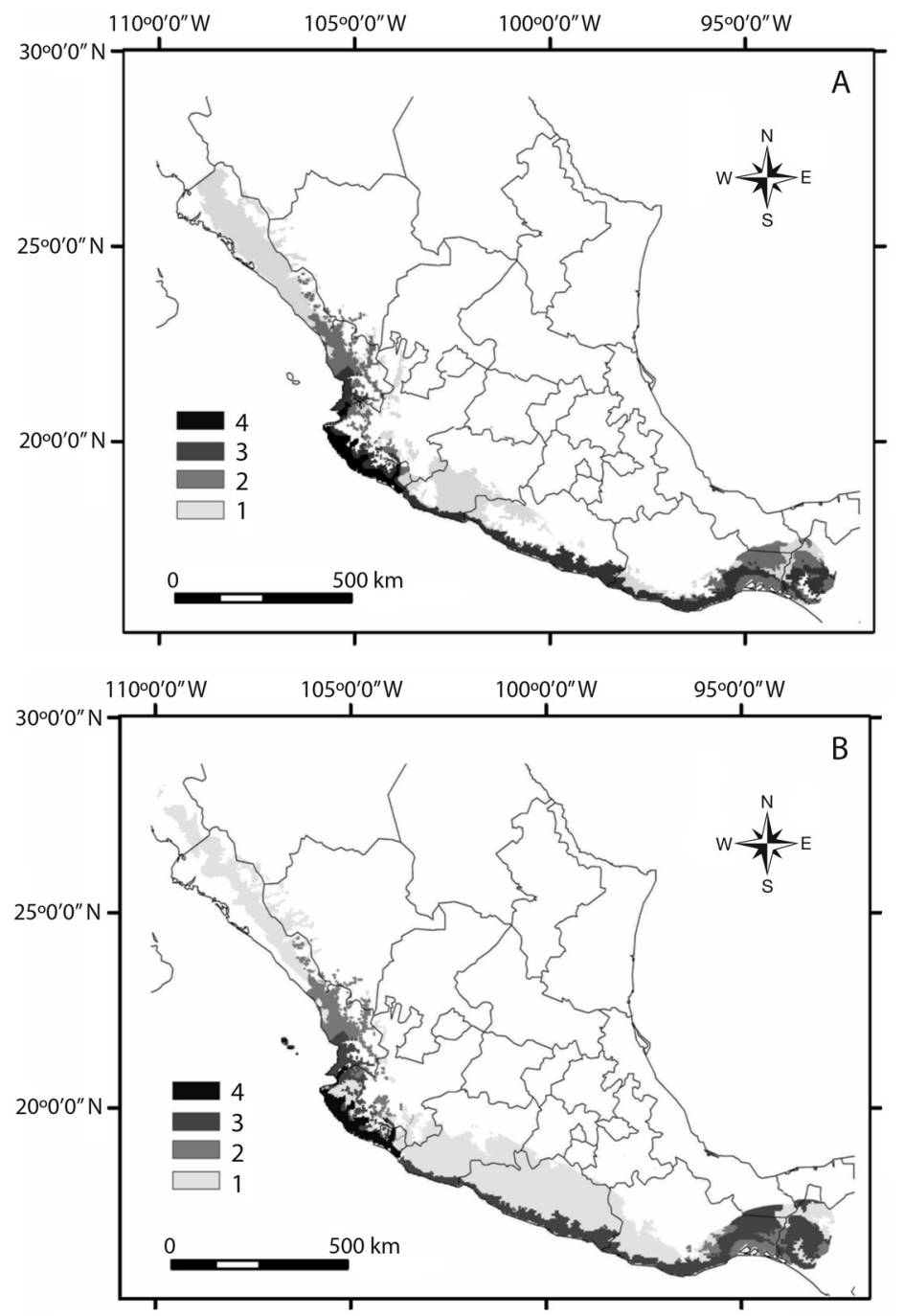

Fig. 5. Correspondencia geográfica entre la distribución potencial de $A$. finschi y la presencia de árboles que sirven de alimento (A) y árboles que sirven para nidificación (B). La gradación de los grises corresponde a la coincidencia en la distribución de la riqueza.

Fig. 5. Geographical overlap between the potential distribution of $A$. finschi and the presence of trees that works as food (A) and trees that works for nesting (B). The grayscale corresponds to the coincidence in the distribution of richness.

se sabe que el porcentaje de pérdida de hábitat de $A$. finschi es bajo en comparación a otras especies (alrededor de un $26 \%$; Ríoz-Muñoz \& Navarro-Sigüenza, 2009). Sin embargo, dada la sensibilidad de la especie a cualquier grado de reducción, puede tener un impacto considerable en su presencia (Renton \& Salinas-Melgoza, 2004). Además, las especies de importancia para el loro corona lila, como las que proveen alimento o sitio de anidación, se corresponden de manera variable y presentan porcentajes de coexistencia menores al $4 \%$, lo que aunado a la sensibilidad de la especie, puede tener impactos negativos en su reproducción (Renton, 2001; Ortega-Rodríguez \& Monterrubio-Rico, 2008).

Si bien históricamente se han reportado varias especies de árboles como de importancia para A.finschi (Renton, 2001; Ortega-Rodríguez 
\& Monterrubio-Rico, 2008), nuestros resultados muestran que la coexistencia geográfica entre los árboles y el loro es muy baja. Lo anterior puede deberse a la escala de los trabajos y a la ubicación. La zona centro de la distribución del loro es la que presenta una mayor coexistencia de las especies vegetales usadas por el loro, dicha coexistencia permitiría a la especie elegir entre los recursos disponibles, lo cual a su vez explicaría los patrones de alimentación y anidación observados (Renton, 2001; OrtegaRodríguez \& Monterrubio-Rico, 2008).

De acuerdo a Ríos-Muñoz y NavarroSigüenza (2009), la mayor pérdida de hábitat para el loro corona lila se ha presentado en las zonas centro y sur de su distribución, siendo el centro el área de mayor abundancia de la especie (Renton \& Iñigo-Elías, 2003), y la zona en la cual encontramos las mejores condiciones climáticas y de disponibilidad de recursos explotados por el loco. Un ejemplo de lo anterior, es la reducción del rango de anidación de la especie en Michoacán, donde ahora sólo ocurre entre los 25 y 600 msnm, ya que en zonas cercanas al nivel del mar, prácticamente no existe cobertura vegetal original (Ortega-Rodríguez \& Monterrubio-Rico, 2008). Además, se sabe que sólo el $2 \%$ de la distribución de la especie se encuentra representada en áreas naturales protegidas (Ríos-Muñoz \& Navarro-Sigüenza, 2009). Asimismo, nosotros encontramos que solo un $\sim 4 \%$ del área de distribución del loro cuenta con la presencia potencial de las principales especies vegetales explotadas por el loro, lo cual sugiere que es necesario tomar medidas urgentes en la conservación de la especie, así como en la evaluación de su dieta y anidación a lo largo de su distribución.

El centro de la distribución de $A$. finschi presenta en un mismo espacio geográfico, las condiciones de las que la especie hace uso durante la temporada seca y la temporada de lluvias, esto puede ser causante de la mayor abundancia de la especie descrita para esta zona (Renton \& Íñigo-Elías, 2003). No obstante, hacia los extremos del rango de distribución, las condiciones no se presentan en un mismo espacio geográfico, lo que en conjunto con la sensibilidad de la especie, podría implicar una menor abundancia respecto a la zona centro. Lo anterior sugiere que las poblaciones extremas (en la zona sur y norte), están sujetas a una mayor presión, que aunado a la pérdida de hábitat en el sur de la distribución (Ríos-Muñoz \& Navarro-Sigüenza, 2009), puede generar extinciones locales, como aparentemente ha sucedido en Oaxaca (Renton \& Íñigo-Elías, 2003).

$\mathrm{Al}$ momento de realizar cualquier planeación para conservar especies que presentan algún tipo de migración y los distintos hábitats de los que hacen uso, es necesario considerar todas las áreas de las cuales las especies hacen uso a lo largo del año. La pérdida de cualquiera de estas áreas de importancia en la estacionalidad de su distribución, disminuirá la capacidad de carga del ambiente, generando presión selectiva en las especies. En el caso de A. finschi, aparentemente sería suficiente con conservar el área central de su distribución; sin embargo, es necesario evaluar la diversidad genética del loro corona lila a lo largo de toda su distribución, ya que las presiones diferenciales pueden estar reduciendo la diversidad génica, que en conjunto con la reducción de su distribución, puede volver a la especie susceptible a cuellos de botella, como pudo haber ocurrido en Oaxaca o en algunos pisos altitudinales donde la especie aparentemente ya no está. Una opción para la conservación de la especie es el establecimiento y protección de corredores biológicos naturales que comuniquen entre sí a las poblaciones, con el fin de aumentar el flujo génico y mejorar la dinámica poblacional. Además, conjugando estos esfuerzos con propuestas de ecoturismo, se puede generar riqueza localmente y con ello ayudar a reducir la extracción de esta y otras especies.

Finalmente, es necesario contextualizar que en este trabajo se presenta un panorama de la distribución macroecológica y geográfica de la especie, incluyendo una perspectiva estacional que permitirá entender las variaciones en la abundancia y conducta migratoria de la especie. Esto, esperamos ayude a mejorar las estrategias de conservación de esta y otras especies 
con patrones estacionales semejantes. Sin embargo, es necesario continuar los esfuerzos con estudios encaminados a evaluar aspectos demográficos, genéticos, así como de cambio climático.

\section{AGRADECIMIENTOS}

Agradecemos a la Dirección General de Investigaciones de la Universidad Veracruzana por la beca de apoyo al Sistema Nacional de Investigadores otorgada a Alejandro Sánchez Barradas con número de personal 43333 y a Cristel Álvarez-Castillo por el apoyo brindado en la edición de las figuras.

\section{RESUMEN}

El loro corona lila (Amazona finschi), es una especie endémica de las tierras bajas de la costa del Pacífico mexicano y está considerada en peligro. Sin embargo, ha sido documentado que la especie presenta migraciones estacionales y altitudinales a lo largo de su rango geográfico, lo que sugiere que su distribución ecológica y temporal permanece incierta. Se modeló la distribución potencial del loro corona lila considerando sus dos principales periodos de actividad: reproductiva y no reproductiva. Para desarrollar modelos de nicho ecológico, se usaron 428 presencias históricas de bases de datos de libre acceso en internet, que fueron combinados con cinco coberturas ambientales: tres climáticas y dos topográficas para cada periodo, mediante el algoritmo de Maxent. Posteriormente transferimos cada modelo estacional al resto de los meses para analizar si los movimientos estacionales de la especie estaban dirigidos por el clima. Se evaluaron las diferencias en las variables ecológicas entre estaciones mediante el uso de una prueba de " $t$ ". Analizamos mediante la superposición de los mapas, la correspondencia geográfica entre la distribución de loro y la de los recursos (plantas), usados por la especie. Se encontró una marcada estacionalidad en la distribución del loro corona lila. La especie presenta una mayor amplitud ecológica en términos de temperatura mínima durante la temporada reproductiva, pero una notable reducción en lo que se refiere a la precipitación. La distribución de los recursos de anidación y alimentación corresponde en gran medida con la distribución del loro corona lila. En el centro del rango de distribución de la especie existe un área donde se presentan las condiciones de las que la especie hace uso en ambas temporadas y en los periodos de transición entre estas. Finalmente, además de las implicaciones en la conservación de la especie, nuestros resultados acerca de la variación estacional en la distribución geográfica y ecológica del loro corona lila, son importantes para la comprensión de la distribución de otras especies, particularmente aquellas que se encuentran asociadas con ambientes altamente estacionales.

Palabras clave: temporada reproductiva, modelado, nicho ecológico, en peligro, anidación, dieta, coexistencia.

\section{REFERENCIAS}

Álvarez, M. (2010). Ecología y distribución potencial de la familia Psittacidae en una zona de transición templado-tropical del bajo Balsas, Michoacán (Tesis de Licenciatura). Universidad Michoacana de San Nicolás de Hidalgo, Hidalgo, México.

Anderson, S. H. (1973). Seasonal variation in forest birds of Western Oregon. Northwest Science, 46, 194-206.

Barve, N. (2008). Tool for Partial-ROC (Biodiversity Institute, Lawrence, $\mathrm{KS}$ ), ver 1.0.

Barve, N., Barve, V., Jiménez-Valverde, A., Lira-Noriega, A., Maher, S. P., Peterson, A. T. \& Villalobos, F. (2011). The crucial role of the accessible area in ecological niche modeling and species distribution modeling. Ecological Modelling, 222, 1810-1819.

Boyle, W. A. (2008). Partial migration in birds: tests of three hypotheses in a tropical lekking frugivore. Journal of Animal Ecology, 77, 1122-1128.

Boyle, W. A. \& Conway, C. J. (2007). Why migrate? A test of the evolutionary precursor hypothesis. American Naturalist, 169, 344-359.

Codesido, M. \& Bilenca, D. (2004). Variación Estacional de un Ensamble de Aves en un Bosque Subtropical Semiárido del Chaco Argentino. Biotropica, $36,544-554$.

Diario Oficial de la Federación (D.O.F.). (2010). Secretaría de Medio Ambiente y Recursos Naturales (SEMARNAT). (Protección ambiental-Especies Nativas de México de flora y fauna silvestres-Categorías de riesgo y especificaciones para su inclusión, exclusión o cambio-Lista de especies en riesgo). México: SEMARNAT.

Environmental Systems Research Institute (ESRI). (2011). ArcGIS Desktop. CA: Redlands.

Forshaw, J. M. (1989). Parrots of the World (3a. ed.). Australia: Lansdowne Editions.

Friedmann, H., Griscom, L. \& Moore, R. T. (1950). Distributional Check-List of the Birds of Mexico. Pacific Coast Avifauna, 29, 1-102.

García-Trejo, E. \& Navarro-Sigüenza, A. (2004). Patrones biogeográficos de la riqueza de especies y el endemismo de la avifauna en el oeste de México. Acta Zoológica Mexicana, 20, 167-185. 
Gómez de Silva, H., Oliveras de Ita, A. \& Medellín, R. A. (2005a). Comisión Nacional para el Conocimiento de la Biodiversidad. (Amazona albifrons). Vertebrados superiores exóticos en México: diversidad, distribución y efectos potenciales). México, Distrito Federal: Instituto de Ecología, UNAM.

Gómez de Silva, H., Oliveras de Ita, A. \& Medellín, R. A. (2005b). Comisión Nacional para el Conocimiento de la Biodiversidad. (Amazona autumnalis). Vertebrados superiores exóticos en México: diversidad, distribución y efectos potenciales). México, Distrito Federal: Instituto de Ecología, UNAM.

Herrera, C. M. (1978). Ecological correlates of residence and non-residence in a Mediterranean passerine bird community. Journal of Animal Ecology, 47, 871-890.

Hijmans, R. J., Cameron, S. E., Parra, J. L., Jones, P. G. \& Jarvis, A. (2005). Very high resolution interpolated climate surfaces for global land areas. International Journal of Climatology, 25, 1965-1978.

Hilty, S. L. (1997). Seasonal distribution of birds at a cloud-forest locality, the Anchicayá Valley, in western Colombia. Ornithological Monographs, 48, 321-343.

Howell, S. \& Webb, S. (1995). A Field Guide to the Birds of Mexico and Northern Central America (1a. ed.). USA: Oxford University Press.

Karr, J. R. (1976). Seasonality, resource availability, and community diversity in tropical bird communities. American Naturalist, 110, 973-994.

La Sorte, F. A., Hochachka, W. M., Farnsworth, A., Sheldon, D., Van Doren, B. M., Fink, D. \& Kelling, S. (2015). Seasonal changes in the altitudinal distribution of nocturnally migrating birds during autumn migration. Royal Society Open Science, 2, 150347.

Leitner, S., Voigt, C. \& Gahr, M. (2001). Seasonal changes in the song pattern of the non-domesticated island canary (Serinus canaria), a field study. Behaviour, 138, 885-904.

Liknes, E. T. \& Swanson, D. L. (2011). Phenotypic flexibility in passerine birds: Seasonal variation of aerobic enzyme activities in skeletal muscle. Journal of Thermal Biology, 36, 430-436.

Loiselle, B. A. \& Blake, J. G. (1991). Resource abundance and temporal variation in fruit eating birds along a wet forest elevational gradient in Costa Rica. Ecology, 72, 180-193.

Loiselle, B. \& Blake, J. (1994). Annual variations in birds and plants of a tropical second growth woodland. The Condor, 96, 368-380.

Lobo, J. M., Jiménez-Valverde, M. \& Real, R. (2008). AUC: a misleading measure of the performance of predictive distribution models. Global Ecology and Biogeography, 17, 141-151.
Malizia, L. R. (2001). Seasonal fluctuations of birds, fruits and flowers in a subtropical forest of Argentina. The Condor, 103, 45-61.

Marín-Togo, M. C., Monterrubio-Rico, T. C., Renton, K., Rubio-Rocha, Y., Macías-Caballero, C., OrtegaRodríguez, J. M. \& Cancino-Murillo, R. (2012). Reduced current distribution of Psittacidae on the Mexican Pacific coast: potential impacts of habitat loss and capture for trade. Biodiversity and Conservation, 21, 451-473.

Masera, O. R., Ordóñez, M. J. \& Dirzo, R. (1997). Carbon emissions from Mexican forests: Current situation and long-term scenarios. Climatic Change, 35, 265-295.

Monterrubio-Rico, T. C., Ortega-Rodríguez, J. M., MarínTogo, M. C., Salinas-Melgoza, A. \& Renton, K. (2009). Nesting habitat of the Lilac-crowned Parrot in a modified Landscape. Biotropica, 41, 361-368.

Nakazawa, Y., Peterson, A. T., Martínez-Meyer, E. \& Navarro-Sigüenza, A. G. (2004). Seasonal niches of neartic-neotropical migratory birds: implications for the evolution of migration. The Auk, 121, 610-618.

Navarro-Sigüenza, A. G., Rebón-Gallardo, M. F., GordilloMartínez, A., Peterson, A. T., Berlanga-García, H. \& Sánchez-González, L. (2014). Biodiversidad de las aves en México. Revista Mexicana de Biodiversidad, $85,476-495$.

Navarro-Sigüenza, A. G. \& Peterson, A. T. (2007). Comisión Nacional para el Conocimiento de la Biodiversidad. 'Amazona finschi (loro corona-lila) residencia permanente. Distribución potencial'. Extraído del proyecto CE015: 'Mapas de las aves de México basados en $W W W$ '. México: Museo de Zoología, Facultad de Ciencias, UNAM \& University of Kansas, Museum of Natural History. Comisión Nacional para el Conocimiento y Uso de la Biodiversidad (CONABIO).

Ortega-Rodríguez, J. M. \& Monterrubio-Rico, T. C. (2008). Características geográficas de la ubicación de nidos del loro corona Lila (Amazona finschi) en la costa del Pacífico en Michoacán, México. Ornitología Neotropical, 19, 427-439.

Papeş, M. (2007). Ecological niche modeling approaches to conservation of endangered and threatened birds in Central and Eastern Europe. Biodiversity Informatics, 4, 14-26.

Parra-Martínez de la, S. M. (2011). Efecto de la variabilidad climática sobre la reproducción del loro corona lila (Amazona finschi) (Tesis de Maestría). Universidad Nacional Autónoma de México, México, Distrito Federal.

Pennington, T. D. \& Sarukhán, J. (2005). Árboles tropicales de México, Manual para la identificación de las principales especies. México, Distrito Federal: Fondo de Cultura Económica. 
Pérez-Moreno, H., Martínez-Meyer, E., Soberón, J. \& Rojas-Soto, O. (2016). Climatic patterns in the establishment of wintering areas by North American migratory birds. Ecology and Evolution, 6, 2022-2033.

Peterson, A. T., Papes, M. \& Soberón, J. (2008). Rethinking receiver operating characteristic analysis applications in ecological niche modeling. Ecological Modelling, 213, 63-72.

Phillips, S. J., Anderson, R. P. \& Schapire, R. E. (2006). Maximum entropy modeling of species geographic distributions. Ecological Modelling, 190, 231-259.

Rabenold, K. N. \& Rabenold, P. P. (1985).Variation in elevational migration, winter segregation, and site tenacity in two subspecies of Dark-eyed Juncos in Southern Appalachians. The Auk, 102, 805-819.

Renton, K. (2001). Lilac-crowned parrot diet and food resource availability: resource tracking by a parrot seed predator. The Condor, 103, 62-69.

Renton, K. (2002). Influence of environmental variability on the growth of Lilac-crowned parrot nestlings. Ibis, 144, 331-339.

Renton, K. (2009). Lilac-crowned parrot (Amazona finschi). Retrieved from http://neotropical.birds.cornell. edu/portal/species/overview?p_p_spp $=23510$

Renton, K. \& Íñigo-Elías, E .E. (2003). Evaluación del estado actual de las poblaciones del loro corona lila (Amazona finschi) en México (Informe final del proyecto AS001). México: Instituto de Biología, UNAM.

Renton, K. \& Salinas-Melgoza, A. (1999). Nesting behavior: of the Lilac-crowned parrot. Wilson Bulletin, $111,488-493$.

Renton, K. \& Salinas-Melgoza, A. (2004). Climatic variability, nest predation, and reproductive output of Lilaccrowned Parrots (Amazona finschi) in tropical dry forest of western Mexico. The Auk, 121, 1214-1225.

Ridgely, R. S. (1981). The current distribution and status of mainland Neotropical parrots. In R. S. Ridgely (Ed.), Conservation of New World parrots (pp. 233-384). School of Forestry and Environmental Studies: Yale University Press.

Ríos-Muñoz, C. A. \& Navarro-Sigüenza, A. G. (2009) Efectos del cambio de uso de suelo en la disponibilidad hipotética de hábitat para los psitácidos de México. Ornitología Neotropical, 20, 491-509.

Robb, G. N., McDonald, R. A., Chamberlain, D. E., Reynolds, S. J., Harrison, T. J. E. \& Bearhop, S. (2008). Winter feeding of birds increases productivity in the subsequent breeding season. Biology Letters, $4,220-223$.

Russello, M. A. \& Amato, G. (2004). A molecular phylogeny of Amazona: implications for Neotropical parrot biogeography, taxonomy, and conservation. Molecular Phylogenetics and Evolution, 30, 421-437.

Rzedowski, J. (1978). La vegetación de México. México: Ed. Limusa.

Salinas-Melgoza, A. A. \& Renton, K. (2005). Seasonal variation in activity patterns of juvenile lilac-crowned parrots in tropical dry forest lilac-crowned parrots in tropical dry forest. Wilson Bulletin, 117, 291-295.

Sarmiento, G. (1972). Ecological and floristic convergence between seasonal plant formations of Tropical and Subtropical South America. Journal of Ecology, $60,367-410$.

Sekercioglu, C. H. (2010). Partial migration in tropical birds: the frontier of movement ecology. Journal of Animal Ecology, 79, 933-936.

Selman, R. G. \& Houston, D. C. (1996). The effect of prebreeding diet on reproductive output in zebra finches. Proceedings of the Royal Society of London B, 263, 1585-1588.

Soberón, J. (2007). Grinnellian and Eltonian niches and geographic distributions of species. Ecology Letters, $10,1115-1123$

Sobral-Marcondes, R., Del-Rio, G., Rego, M. A. \& Silveira, L. F. (2014). Geographic and seasonal distribution of a little-known Brazilian endemic rail (Aramides mangle) inferred from occurrence records and ecological niche modeling. The Wilson Journal of Ornithology, 126, 663-672.

Sorensen, M. C., Hipfner, J. M., Kyser, T. K. \& Norris, D. R. (2009). Carry-over effects in a Pacific seabird: Stable isotope evidence that pre-breeding diet quality influences reproductive success. Journal of Animal Ecology, 78, 460-467.

Stiles, G. (1978). El ciclo anual en una comunidad coadaptada de colibríes y flores en el bosque tropical muy húmedo de Costa Rica. Revista de Biología Tropical, $27,75-102$

Stotz, D. F., Fitzpatrick, J. W., Parker, T. A. \& Moskovits, D. K. (1996). Neotropical birds: Ecology \& conservation (1a. ed.). Chicago, USA: University of Chicago.

Trejo, I. \& Dirzo, R. (2000). Deforestation of seasonally dry tropical forest a national and local analysis in Mexico. Biological Conservation, 94, 133-142.

United States Geological Survey (USGS). (2005). USGS Hydrolk Elevation Derivative Database. U. S. Department of the Interior, U. S. Geological Survey. Retrieved from http://edc.usgs.gov/products/elevation/gtopo30/hydro/index.html

Wiens, J. A. (1989). The ecology of bird communities (Vol. 1). Foundations and patterns. Cambridge, England: Cambridge University Press. 